\title{
Ampiginous Choroiditis
}

\author{
Joumany Brahim Salem, Imane Tarib, Mahdi Khammaily, Sidi Dahi, Youssoufou Souley Abdoul \\ salam, Rachid Zarrouk, Yassine Mouzari, Fouad El Asri, Karim Reda, and Abdelbarre Oubaaz
}

\begin{abstract}
Ampiginous choroiditis is a rare inflammatory disease that progressively alters the chorio-capillary, pigmented epithelium (PE) and neurosensory retina. We report the case of a 43-year-old patient with decreased visual acuity at finger count (CF) in both eyes, with multiple yellowish-white lesions of varying size, mostly in the peri-papillary region
\end{abstract}

Index Terms - Ampiginous choroiditis, Relentless placoid maculopathy, Serpiginous choroiditis, Placoid pigmentepitheliopathy.

\section{INTRODUCTION}

Ampiginous choroiditis is a rare, inflammatory disease that appears to be related to localized choroidal ischemia. This condition progressively alters the chorio-capillary, the retinal pigmented epithelium (RPE) and the neurosensory retina, thus often resulting in a very low visual acuity.Its management remains poorly defined until today.

\section{PATIENTS AND OBSERVATIONS}

We report the case of a 43-year-old patient, with no significant medical history, who consults for a gradual decrease in visual acuity in both eyes, without redness, pain or photophobia. The ophthalmological examination found visual acuity to be count fingers at 1 meter that could not be improved after optical correction in both eyes. The examination of the appendices of the eye is without particularities; Examination of the anterior segment found a clear cornea without retro-descemetic precipitate, an optically empty anterior chamber, a normo-trophic normocolored iris, a transparent lens and vitreous in both eyes. The fundus of the right eye shows multiple peripapillary yellowish white lesions of variable size and a pre-macular fibrosis, and in the left eye we found a pre-macular hemorrhage with peripapillary atrophy and multiple whiteyellowish peripapillary lesions. (Figure 1a,b)
Published on May 11, 2020.

Authors are with Mohammed V Military Instructional Hospital, Faculty of Medicine and Pharmacy, Mohammed V University, Rabat, Morocco.

(corresponding e-mail: joumanybss@gmail.com)

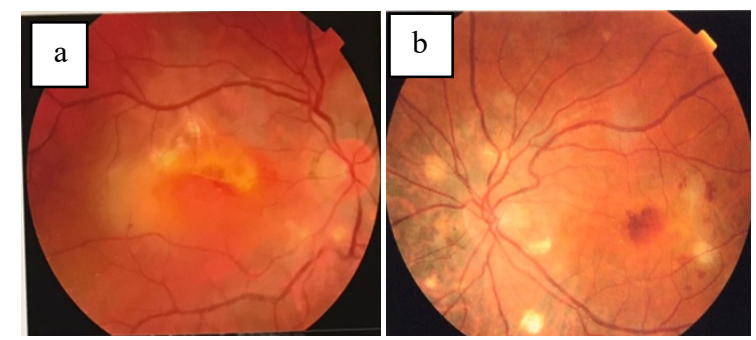

Figure (1a) OD Retinal photography: Multiple yellowish white lesions of variable size predominantly peripapillary with pre-macular fibrosis. Figure (1b) OG Retinal photography: Multiple yellowish white lesions of variable size predominantly peripapillary with pre-macular hemorrhage.
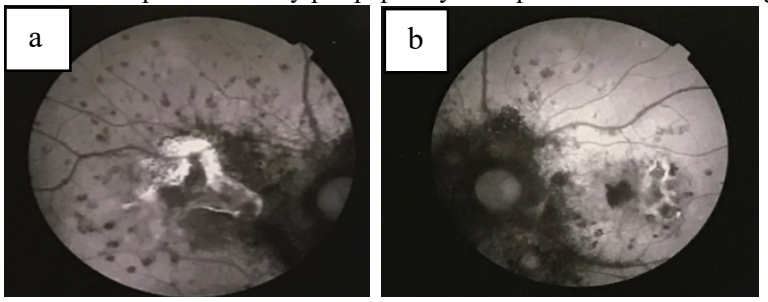

Figure (2a) OD Auto-fluorescence pictures: hypo-auto-fluorescence of peripapillary lesions and hyper-auto-fluorescence of perimacular lesions Figure (2b) OG Auto-fluorescence pictures: hypo-auto-fluorescence of lesions in the peripapillary and hyper-auto-fluorescence in the perimacular.

On fluorescein angiograms, hypo-fluorescence of the lesions is noted at an early stage with a late weak impregnation. (Figure 3)

Macular OCT in both eyes shows intra retinal edema with deposits of hyper-reflective material in the outer layers with alteration of the RPE, the IS / OS line of photoreceptors as well as dilation of the chorio-capillary. (Figure 4)
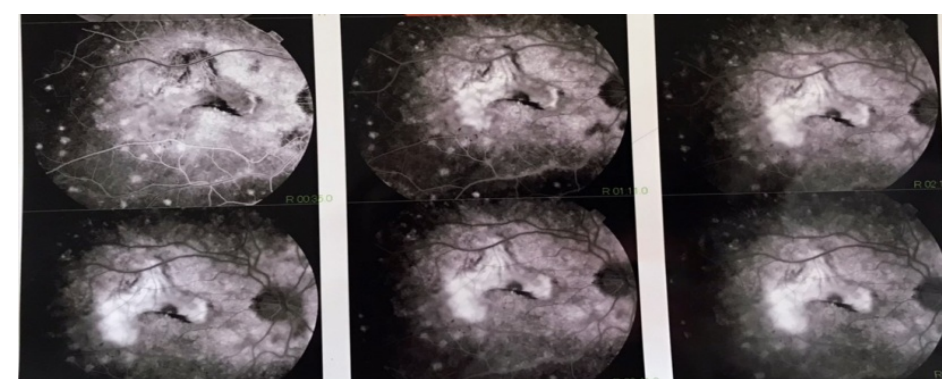

Figure (3a) OD Fluorescein angiograms: hypofluorescence of lesions at early time with weak impregnation at late time
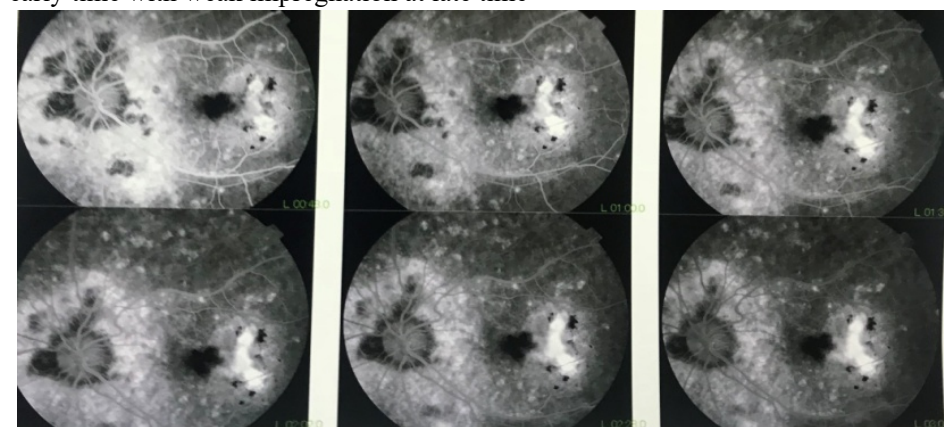
Figure (3b) OG Fluorescein angiograms: hypofluorescence of lesions at an early stage with weak impregnation at a late stage

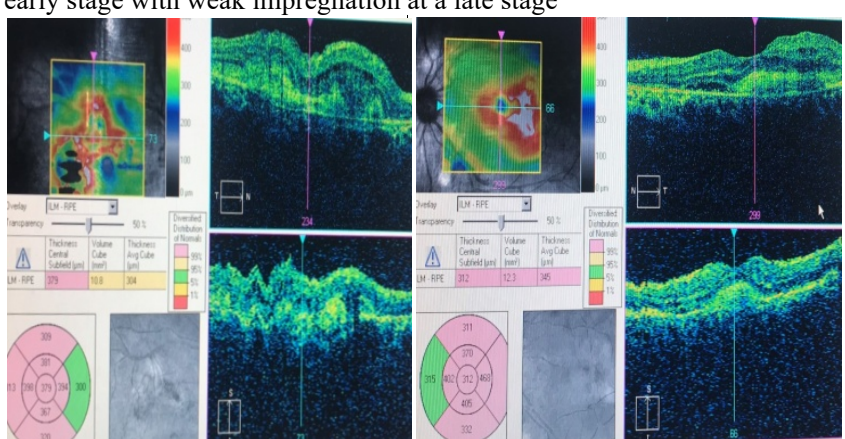

Figure (4a) OD: macular OCT intra retinal edema, hyper-reflectivity of the outer layers with alteration of the $\mathrm{PE}$.

Figure (4b) OG: macular OCT intra retinal edema, hyper-reflectivity of the outer layers with alteration of the $\mathrm{PE}$

The rest of the somatic examination is without particularities.

The results of tuberculosis and viral serologies, syphilitic and Lyme disease returned negative.The phosphocalcic balance, the conversion enzyme and the immunological balance was normal.Given the patient's age, the presence of severe visual impairment, the site of the lesions and the absence of other associated signs, the diagnosis of ampiginous choroiditis was retained.

The patient received a corticosteroid bolus 3 days in a row and then received an oral relay with an intra-vitreous (IVT) injection of anti VEGF in both eyes.

The evolution was marked by the improvement in visual acuity which increased to $1 / 10$ in the right eye and $4 / 10$ in the left eye.

\section{DISCUSSION}

Ampiginous choroiditis has recently been described as a clinical entity combining the characteristics of multiple epitheliopathy (APMPPE) and serpiginous choroiditis in relatively young, male patients. But it is differentiated by its evolution and its visual prognosis, hence the importance of differentiating them (1).

APMPPE is a bilateral, asymmetrical condition occurring following a flu-like syndrome, its progression is towards a spontaneous regression with few recurrences and its visual prognosis is generally good (2).

Serpiginous choroiditis (SC) is a rare disease $(5 \%)$, of an unknown cause and potentially blinding (3). It mainly affects middle-aged patients with a male predominance. It installs gradually, without prodrome, with a significant and often permanent decrease in vision. The lesions in the SC appear around the papilla, most often in the vicinity of scar lesions and extend centrifugally from the papilla towards the macula or the retinal periphery by branching. SC can be classified into three forms: peripapillary, macular and ampiginous (4). Ampiginous choroiditis is characterized by lesions of smaller size than the serpigineuse of about half a disc with recurrences which are not necessarily on the edges of old lesions as in SC (5).

Fuoroceinangigraphy (FA) of the active lesions shows hypo-fluorescence at early times with late impregnation by choroidal hypo-perfusion; and in case of scar lesions hypofluorescence surrounded by a hyperfluorescent border. The disappearance of this border marks the progressive recovery of the disease (sign of Bernard). Early diffusion indicates the appearance of sub-retinal neovascularization (6).In auto-fluorescence, the active lesions are most often hyper-auto-fluorescent while the scar lesions are hypo-autofluorescent by destruction of the RPE cells; hence the interest of these pictures for monitoring the disease (6).

Macular OCT shows at the initial stage a high reflectivity of the external retina and of the RPE with conservation of the internal retina. Scarring results in loss of the ellipsoid area with hyper-reflectivity of the choroid, loss of RPE and external segments of the photoreceptors (6).

Visual impairment in the ampiginous form remains of poor prognosis and this would be due to the central location of the lesions, the frequency of the choroidal neovessels (CNV) and the sub-retinal fibrosis which develops fairly quickly despite aggressive treatment (5).

Several therapeutic options have been proposed but the results of the treatments remain difficult to assess due to the rarity and variability of the course of the disease. Corticosteroids are used in the acute phase of the disease, whether or not followed by immunosuppressive therapy depending on the course or recurrence after cessation. Corticosteroid-cyclosporine-azathioprine combinations (triple therapy) or cyclosporine and other immunosuppressants such as mycophenolate mofetil or azathioprine have also been proposed with controversial results. (6)

In cae of $\mathrm{CNV}$, the Anti-VEGF treatment is a good option, and usually 1 to 4 injections are necessary to stop the exudation (7).

\section{CONCLUSION}

Ampiginous choroiditis is a choroiditis which affects young people and which progresses to chorio-retinal atrophy, hence its severity through its potentially blinding effect. The causes of this disease are poorly defined and its management remains difficult.

\section{REFERENCES}

[1] Mansour AM, Jampol LM, Packo KH, Hrisomalos NF. Macular serpiginous choroiditis. Retina Phila Pa. 1988;8(2):125-31.

[2] Hardy RA, Schatz H. Macular geographic helicoid choroidopathy. Arch Ophthalmol Chic Ill 1960. sept 1987;105(9):1237-42.

[3] Lim W-K, Buggage RR, Nussenblatt RB. Serpiginous choroiditis. Surv Ophthalmol. juin 2005;50(3):231-44

[4] Nazari Khanamiri H, Rao NA. Serpiginous choroiditis and infectious multifocal serpiginoid choroiditis. SurvOphthalmol. juin 2013;58(3):203-32.

[5] Amer R, Florescu T. Optical coherence tomography in relentless placoid chorioretinitis. Clin Experiment Ophthalmol. mai 2008;36(4):388-90.

[6] Saleh M. Placoid pigment epitheliopathy and serpiginous choroiditis. J Fr Ophtalmol. févr 2020;43(2):e55-66.

[7] Song M-H, Roh Y-J. Intravitreal ranibizumab for choroidal neovascularisation in serpiginous choroiditis. Eye. sept 2009;23(9):1873-5. 


\section{Joumany Brahim Salem}

e-mail: joumanybss@gmail.com

Hassan 2 University of Casablanca, Morocco

Institution: Department of Ophthalmology,

Mohammed V Military Instructional Hospital,

Faculty of Medicine and Pharmacy Mohammed V University,

Rabat, Morocco.

\section{Imane Tarib}

e-mail: itarib@gmail.com

Institution: Department of Ophthalmology,

Mohammed V Military Instructional Hospital,

Faculty of Medicine and Pharmacy Mohammed V University,

Rabat, Morocco.

Mahdi Khammaily

e-mail: mehdi.khamaily@gmail.com

Institution: Department of Ophthalmology,

Mohammed V Military Instructional Hospital,

Faculty of Medicine and Pharmacy Mohammed V University,

Rabat, Morocco

\section{Sidi Dahi}

e-mail: dahisidi2014@gmail.com

Institution: Department of Ophthalmology,

Mohammed V Military Instructional Hospital,

Faculty of Medicine and Pharmacy Mohammed V University,

Rabat, Morocco.

\section{Yassine Mouzari}

e-mail: ys.mouzari@gmail.com

Institution: Department of Ophthalmology,

Mohammed V Military Instructional Hospital,

Faculty of Medicine and Pharmacy Mohammed V University,

Rabat, Morocco.

\section{Fouad El Asri}

e-mail: elasri 76@hotmail.com

Institution: Department of Ophthalmology,

Mohammed V Military Instructional Hospital,

Faculty of Medicine and Pharmacy Mohammed V University,

Rabat, Morocco.

\section{Karim Reda}

e-mail: mkarimreda@gmail.com

Institution: Department of Ophthalmology,

Mohammed V Military Instructional Hospital,

Faculty of Medicine and Pharmacy Mohammed V University,

Rabat, Morocco.

\section{Abdelbarre Oubaaz}

e-mail: aoubaaz@gmail.com

Institution: Department of Ophthalmology,

Mohammed V Military Instructional Hospital,

Faculty of Medicine and Pharmacy Mohammed V University,

Rabat, Morocco. 